\title{
Light-efficient augmented reality 3D display using highly transparent retro-reflective screen
}

\author{
ShOAIB R SOOMRO, ${ }^{1, *}$ HAKAN UREY, ${ }^{1}$ \\ ${ }^{1}$ Koc University, Electrical Engineering Department, Optical Microsystems Laboratory, Sariyer, Istanbul, Turkey, \\ *Corresponding author: ssoomro13@ku.edu.tr
}

Received XX Month XXXX; revised XX Month, XXXX; accepted XX Month XXXX; posted XX Month XXXX (Doc. ID XXXXX); published XX Month XXXX

\begin{abstract}
We propose and demonstrate a light efficient 3D display using a highly transparent desktop-size augmented reality screen. The display consists of a specially designed transparent retro-reflective screen and a pair of low power picoprojectors positioned close to the viewer's eyes to provide stereo views. The transfer screen is an optically clear sheet partially patterned with retro-reflective microspheres for high optical gain. The retro-reflective material buried in the screen reflect incident light back towards the projectors with narrow scattering angle and facilitates the viewer to perceive a very bright content. The tabletop prototype mainly consists of an in-house fabricated large AR screen $\left(60 \times 40 \mathrm{~cm}^{2}\right)$ and a pair of laser scanning 30 lumen pico-projectors. The display is tested for different viewing configurations, different display parameters such as retro-reflective coefficient, eye-box size, polarization maintainability, stereo crosstalk and brightness. The AR prototype display provides $\mathbf{7 5 \%}$ optical transparency, exceptional brightness (up to $1000 \mathrm{~cd} / \mathrm{m}^{2}$ when viewed through beam-splitters and $350 \mathrm{~cd} / \mathrm{m}^{2}$ with bare eyes) and negligible crosstalk in 3D mode $(<5 \%$ and $<1 \%$ when viewed through beam-splitters and polarizers respectively) for the working distance of up to 2 meters.
\end{abstract}

OCIS codes: (120.2040) Displays; (230.0230) Optical devices; (220.4830) Systems design.

http://dx.doi.org/10.1364/AO.99.099999

\section{INTRODUCTION}

Augmented reality 3D displays have been widely explored for their ability to blend virtual and real word and can be used for variety of applications including entertainment, medical visualization and education. Most of the augmented reality systems are based on one more image sources and optically transparent surfaces [1-7]. Several techniques have been proposed to realize the augmented reality displays, where most of the effort has been focused on the development of near-to-eye augmented reality displays [3-4]. A typical near-to-eye augmented reality display utilizes image projector or micro-display as source and holographic optical elements (HOEs) or half silvered mirror together with the related optical components to relay the image to eye. The 3D capability is added by providing separate views to each eye. With the availability of high resolution micro-displays, the current nearto eye display can produce decent image quality and resolution, but often involve bulky optics and have limited field of view. An alternative approach uses image projectors together with distant transfer screens or real-objects to overlay virtual content [5-8], which includes the integral imaging [5], holographic recorded micro-lens arrays [6], partially reflective or notch coated micro-lens arrays [7] and plasmonic nanocubes [8] based AR displays. The use of additional tunable transmittance liquid crystal film to increase the ambient contrast in wearable augmented reality display have also been demonstrated [9]. However, such displays are hard to fabricate in large size and suffer from lower transparency and/or brightness. The retro-reflective materials (typically used for road safety applications) have also been demonstrated as transfer screen for head mounted projection based bright personalized displays [10-12]. However, due to the nontransparent nature of retro-reflective materials, they haven't been explored as augmented reality displays.

In this paper, we present an augmented reality 3D display, which can provide bright 3D content together with higher optical transparency. The foundation of our display is based on low power mobile projectors and a special see-through screen partially filled with retro-reflective microspheres. The retro-reflective nature of screen delivers lightefficiency and separates stereo views by reflecting incident light back towards source with minimum scattering, while partial filling of retroreflective material provides optical transparency needed for augmented reality applications. In [13], we focused on the optical design and presented the fabrication flow of the transparent retro-reflective screen. This paper focus on the design, implementation and evaluation of large size light efficient augmented reality 3D display using transparent retro-reflective screen. In Section 2, we introduce the concept and working mechanism of the proposed display. Our experimental porotype is presented in Section 3. The retro-reflective and polarization characteristics of display are discussed in Section 4, while display eye-box analysis and experiment results on stereo crosstalk and display brightness are discussed in Section 5 .

\section{CONCEPT OF THE DISPLAY}

The proposed display consists of two pico-projectors and a see-through retro-reflective screen as illustrated in Fig. 1. The retro-reflective material deposited on the screen reflects incident light back towards 
source in the shape of narrow angular cone providing the peak reflectance at the exit pupil of the projector. The projectors are placed close to the viewer's head and the horizontal baseline distance between the projectors is matched with the inter-pupillary distance (IPD) of viewer. The stereo content is projected on see-through screen using the projector pair placed at distance $d$ from the screen, where each projector illuminates the screen with one of the two channels of a stereo 3D content. The light from each projector is turned back and provides bright content to the eye positioned close to associated projector. By utilizing the high contrast of laser pico-projectors, the floating 3D content is displayed by projecting the content with back background.

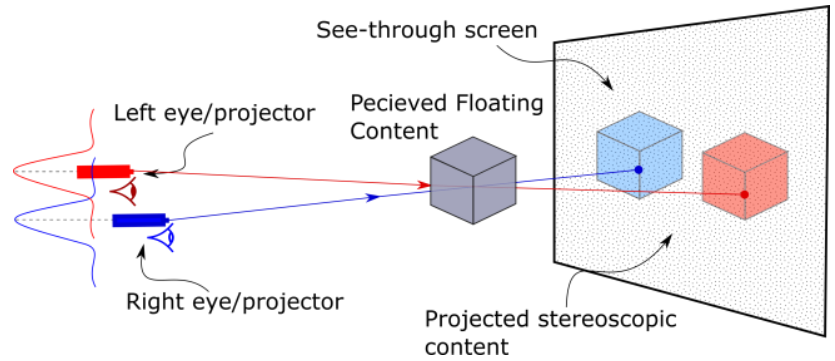

Fig. 1. Illustrates the concept of the display, a projector pair illuminates screen with stereoscopic content, the light from each projector is returned to associated projector and perceived by eye closer to the projector.

We use a specially designed and in-house fabricated transparent retro-reflective surface as transfer screen [11]. The screen is an optically clear sheet consisting of finely patterned retro-reflective microspheres. Since a full retro-reflective surface (i.e. retro-fabric, retro-sticker sheet) can provide higher light efficiency $[14,15]$, we optimize the amount of transparency and retro-reflectivity by partially depositing retroreflective glass microspheres on the clear substrate as square shaped tiny islands. The smaller size of individual islands smooths pattern features and makes it irresolvable by human eye at working distance from screen [13]. To balance the amount of brightness and optical transparency, the $25 \%$ of total screen area was filled with the microspheres, while $75 \%$ of screen area was left see-through.

\section{EXPERIMENTAL PROTOTYPE}

The tabletop experimental prototype was built to demonstrate the augmented reality 3D display as shown in Fig. 2(a). Our setup consisted of two 30 lumen laser sourced Sony pico-projectors [16] and an inhouse fabricated see-through screen. A pair of 3D printed housings together with folding mirror/beam-splitters were used to minimize the distance between eye and projector. Each projector has the resolution of $1280 \times 720$ pixels (720p HD) and covers the $42^{\circ} \times 24^{\circ}$ field of view of each eye. The see-through screen was placed within the distance of $d=50-200 \mathrm{~cm}$ from viewer and the display was tested under normal indoor ambient lighting. The horizontal distance between projectors' exit pupil was varied to match the IPD distance of specific viewer, while an average IPD distance of $6.5 \mathrm{~cm}$ was used during display characterization. The minimum achievable gap $(s)$ between eye and projector was $2 \mathrm{~cm}$ and $0.5 \mathrm{~cm}$ for folding mirrors and beam-splitters respectively.

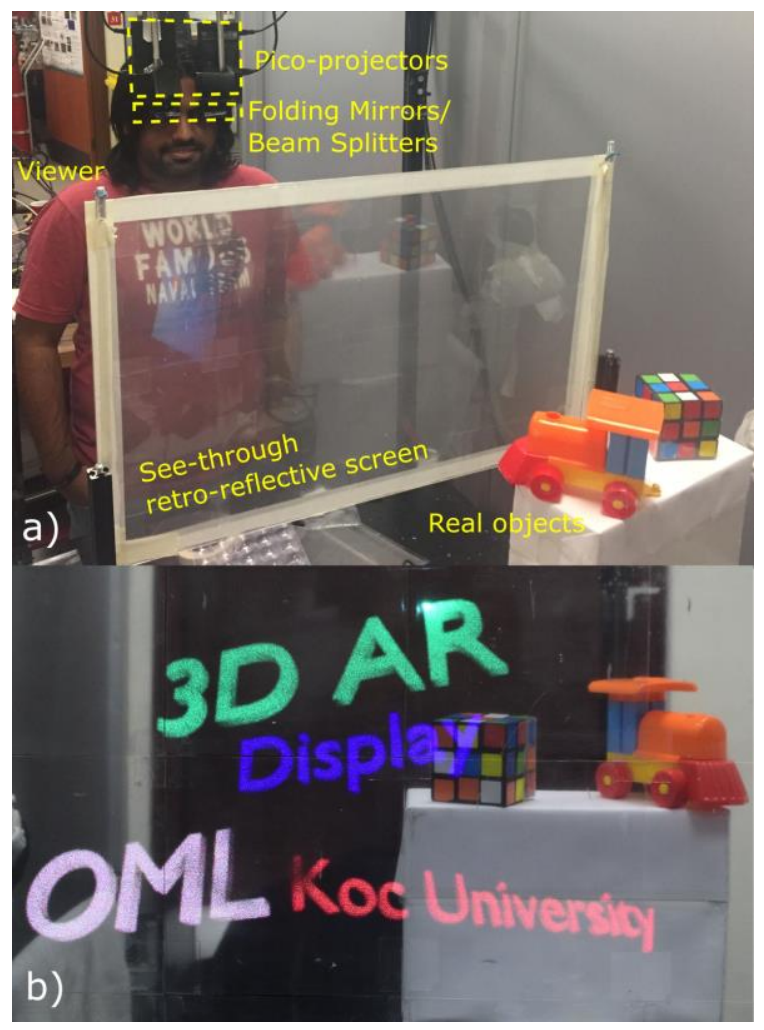

Fig. 2. (a) Shows the desktop size experimental prototype (b) Shows the content as projected using one of the two projectors and perceived by eye closer to associated projector.

The screen fabrication involved the use of half-shellaluminum coated glass microspheres, where an acrylic sheet with thin adhesive coating was used as base substrate and the retro-reflective microspheres were patterned and deposited using a negative shadow mask made of stainless steel. The full-size screen $\left(60 \times 40 \mathrm{~cm}^{2}\right)$ shown in Fig. $2(\mathrm{~b})$ was constructed by tiling together multiple segments of fabricated screen on clear Plexiglas sheet, where size of each screen segment was $16 \times 16 \mathrm{~cm}^{2}$. To have full field overlap between the projected images, both projectors were tilted inward slightly, which introduced the keystone distortion. To provide comfortable viewing experience, the distortion was corrected by projecting a checkerboard image on both projectors and adjusting the horizontal keystone manually.

\section{RETRO-REFLECTIVE AND POLARIZATION CHARACTERISTICS}

Since, the proposed display uses the retro-reflective feature for image display, the quality and performance of the display depend on the retroreflective characteristics of the screen and the configuration of image source with respect to screen. The retro-reflectivity of screen was determined by measuring the retro-reflective coefficient (intensity of retro-reflected light and the angular width of retro-reflective cone).

The retro-reflective coefficient was measured with a photometric setup as reported in [13], where a sample of screen was illuminated with 633 $\mathrm{nm}$ collimated laser beam and amount of retro-reflected light as function of viewing angle was measured. Figure 3 shows the measured retro-reflective coefficient as function of angle between eye and projector with respect to the screen. The photometric setup contained a small size detector subtending the solid angle of $50 \mu \mathrm{sr}$ with the screen. The coefficient value in Fig. 3 shows the amount of light returned within that small solid angle at different angular positions and a maximum of $0.35 \%$ of input light is returned at $0 \mathrm{mrad}$, which is the exit pupil location 
of the source/projector. The measured retro-reflective coefficient is only about 8 times lower than the bestavailable retroreflector (i.e. retroreflective fabric). Factor of 4 reduction is due to the fill factor (screen is $75 \%$ transmissive and 25\% retro-reflective) and additional factor of 2 is due to manufacturing process and will be improved in the future by properly orienting the microspheres on the substrate. Compared to a typical projection screen (i.e. diffused surfaces, which scatter light within the solid angle of $\pi$ steradian), the proposed screen provides increased light efficiency, which is evident by the higher brightness offered by screen as discussed later in Section 5 .

The coefficient value is proportionally increased as the angle between viewer's eye and the projector is decreased. The retroreflective cone also shows the diffraction orders due to the aperture diffraction of microspheres [12]. The aperture diffraction affects the angular width and peak coefficient value of retro-reflective cone. The smaller microspheres result wider retro-reflective cone, while larger microspheres (such as the ones used in airport runways) create extremely narrow angular cone. Since the size of microspheres is at least 10 times smaller $(35-45 \mu \mathrm{m})$ than the projected pixel size $(>400 \mu \mathrm{m})$ the image quality is not affected. A constant coefficient value was observed outside the $\pm 50 \mathrm{mrad}$, which shows the diffused scattering due to geometric and material imperfections of microspheres and substrate.

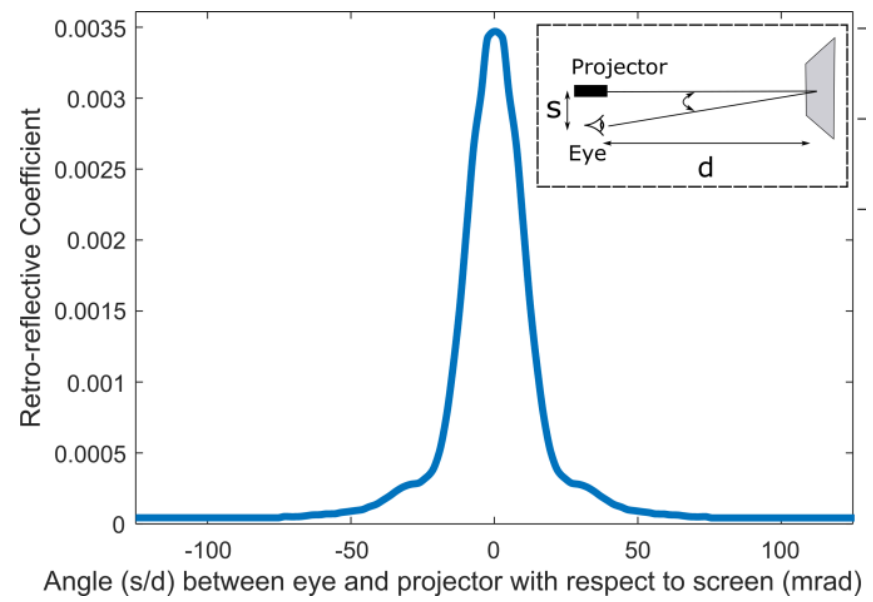

Fig. 3. Shows the experimentally measured retro-reflective coefficient, the microspheres buried in screen create a $100 \mathrm{mrad}$ wide retroreflective cone, the diffraction minima are observed due to the aperture diffraction of microspheres.

Due to the high refractive index of glass microspheres and back aluminum coating, the incident ray is refracted at air-microsphere interfaceand goes though specular reflection ataluminum-microsphere interface. The specular reflection and metal coating facilitates the incident light to preserve its polarization after going through retroreflection. We measured the ability of screen to preserve polarization by using a photometric setup (Fig. 4(a) inset). We illuminated the screen with a linear horizontal polarized light and captured the retro-reflected light using the detector positioned at $0 \mathrm{mr}$ angle. The polarization maintainability was further measured by placing an analyzer (linear polarizer film) before the detector and changing its transmission axis orientation. Figure 4(a) shows the normalized intensity when the analyzer was rotated between $0-90^{\circ}$ with respect to polarization direction of incident light. The figure shows the maximum light intensity when the angle between polarization direction of incident light and transmission axis of analyzer is same. Due to the retained polarization after retro-reflection, the light intensity is decreased as the orientation of analyzer is changed. The normalized intensity of $<2 \%$ in detected at $90^{\circ}$ orientation, due to the amount of light leakage in analyzer. Figure 4(b) and 4(c) show the photos of illuminated screen captured with camera placed next to projector when, the polarizer placed in front of camera is oriented in horizontal and vertical directions respectively.
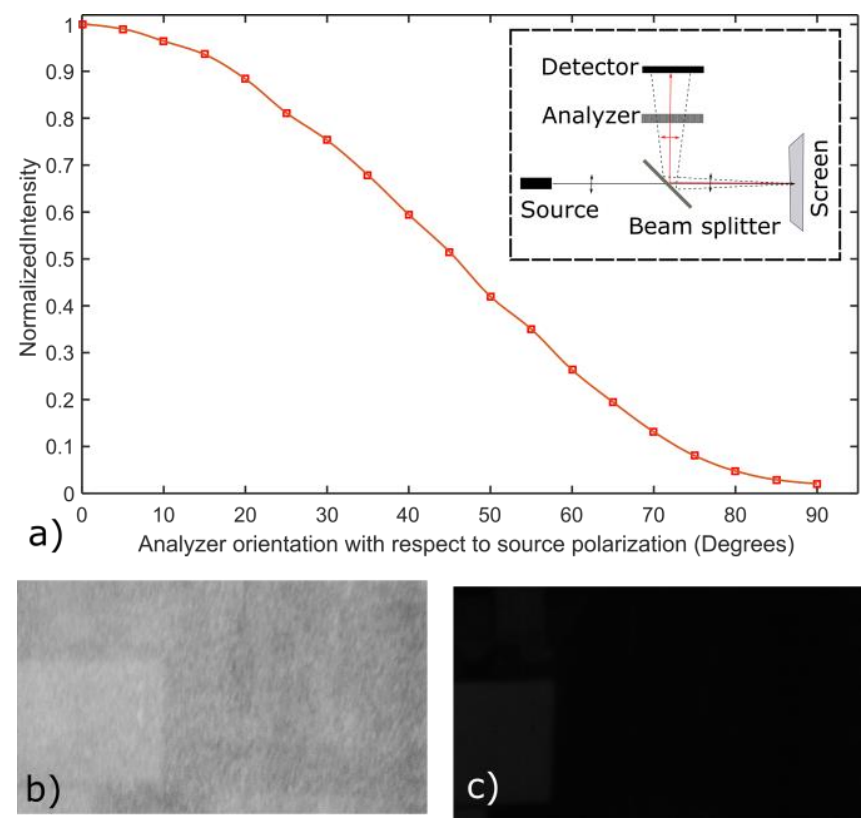

Fig. 4. Shows the polarization maintaining ability of see-through retroreflective screen, a) Normalized captured intensity as a function of polarization orientation measured with a photometric setup, b) and c) show the screen surface illuminated with horizontally polarized light and captured by placing a horizontal polarizer (b) and, vertical polarizer (c) in front of camera. Note that the bright rectangular object in the figures is an incoherent illuminated object behind the screen.

\section{RESULTS AND DISCUSSIONS}

\section{A. Eye-box Analysis}

As shown in Fig. 3, the intensity distribution of retro-reflective cone was measured in only one direction. Since, the retro-reflective cone is circularly symmetrical about $0 \mathrm{mr}$ angle, the $2 \mathrm{D}$ representation of retroreflective cone was obtained by circularly rotating the right side of the retro-reflective cone. The retro-reflective cone has angular spread and actual eye-box size for each eye and overlap between left and right eyebox depend on the angular width of the retro-reflective cone and distance between viewer/projector and the screen. For a defined eyeprojector gap, as the distance between screen and viewer/projector changes, the size and amount of overlap between left and right eye box is changed, which in result changes the stereo crosstalk and perceived brightness.

To minimize the eye-box overlap and eye-to-projector distance, we tested three different viewing configurations as illustrated in Fig. 5, where the screen is viewed, 1) with bare eyes below each projector, 2) through additional polarizer films in front of eyes and,3) through beamsplitters. 


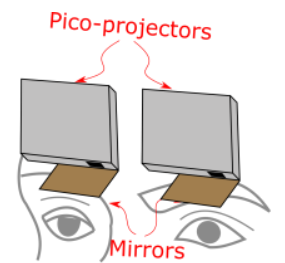

a)

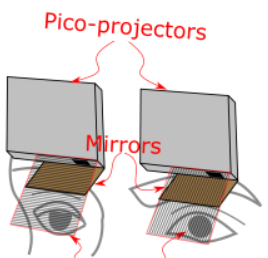

b) Polarizer film

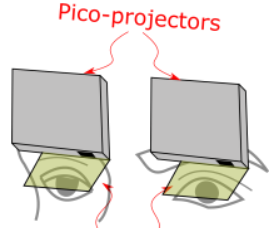

c) Beam splitters

Fig. 5. Illustrates the different eye-projector configurations, (a) bare eye configuration, where projection beam is folded towards screen using mirrors, (b) polarizer based configuration, where additional polarizer films are used to separate two views and, (c) Beam-splitters based configuration, where eye-to projector gap is minimized.

Figure 6 shows the normalized coefficient values, eye-box size and overlap for all three configurations at $100 \mathrm{~cm}$ from the screen. The location of left/right projectors and eyes are $P_{L}, P_{R}$ and $E_{L}, E_{R}$ respectively. For each eye, the distance to closer and farther projector is $s$ and $u$ respectively.
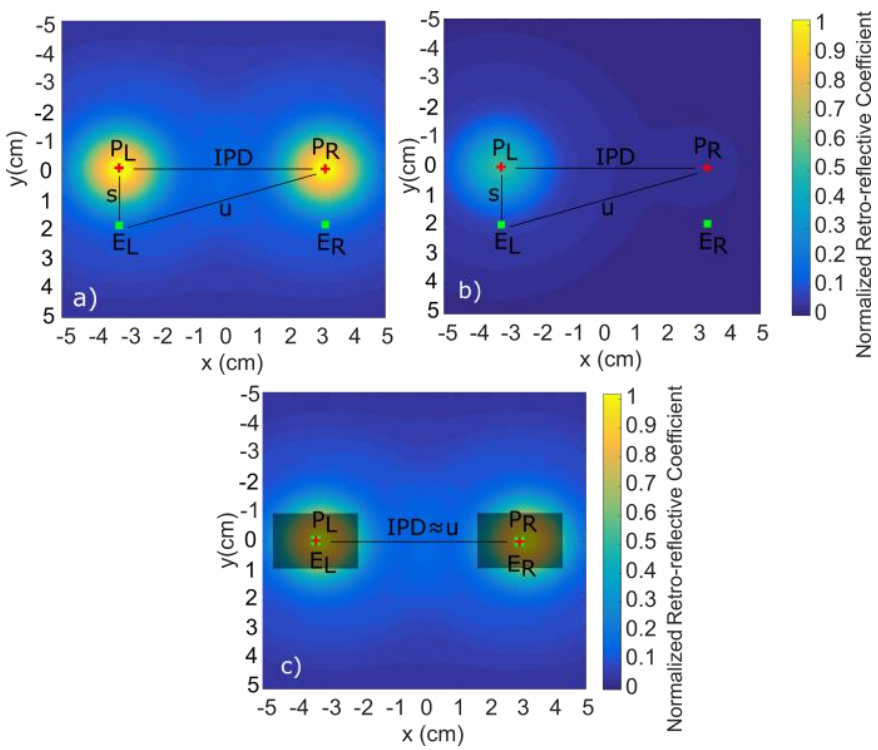

Fig. 6. Shows the eye-box size and overlap for different eye-projector settings at distance of $100 \mathrm{~cm}$ from screen, a) Screen viewed with bare eyes, b) Projectors are polarized in different directions having matching polarizer in front of eyes and, c) 50/50 beam splitters used to align respective eye and projector.

For the bare eye configuration as shown in Fig. 6(a), we use $3 \times 3 \mathrm{~cm}^{2}$ mirrors oriented at $45^{\circ}$ to bend the projector light towards the screen and the viewer's eyes are positioned below the mirrors. The presence of mirrors restricts the closeness of eye and the projector and a minimum gap of $s=2 \mathrm{~cm}$ is achieved. The bare eye configuration provides significant brightness and facilitates ergonomic comfort, but results higher stereo crosstalk due to the distance dependent eye-box size as discussed in the following section.

In the next configuration, we utilize the polarization maintaining property of the screen by cross polarizing the projectors light and placing matching polarizer films in front of eyes. Figure 6(b) shows the eye-box and normalized coefficient for left eye, when viewed through polarizer. The polarization difference between left and right projectors effectively separates both views and facilitates minimum crosstalk.

The eye-projector gap is reduced by replacing the folding mirrors with 50/50 beam-splitters. As shown in Fig. 6(c), the use of beam splitter eliminates the distance between eye and projector and helps to

align the eye with the exit pupil of respective projector. The beam splitter enables the eye to lie at the peak of retro-reflective cone, while the overall coefficient value is affected by factor of four due to the beam splitter.

\section{B. Stereo Crosstalk}

As discussed previously, for each configuration, the eye closer to projector perceives high brightness than the farther eye due the higher coefficient value. Considering the eye-box analysis in Fig. 6 and retroreflective coefficient values, the crosstalk for the left eye can be expressed as:

$$
\text { Crosstalk }_{L}=\frac{C_{R}\left(\frac{u}{d}\right)}{C_{L}\left(\frac{s}{d}\right)} \times 100 \%
$$

Where $C_{R}(u / d)$ and $C_{L}(s / d)$ are the coefficient value of right and left projectors at the viewing angle of $\mathrm{u} / \mathrm{d}$ and $\mathrm{s} / \mathrm{d}$ respectively. The values of $\mathrm{s}$ and $\mathrm{u}$ depends on the eye-projector distance, $\mathrm{s}=2 \mathrm{~cm}$ and $u=\sqrt{s^{2}+I P D^{2}} \mathrm{~cm}$ are used for bare eye and polarized projectors configurations, while $s<0.5 \mathrm{~cm}$ and $u \approx$ IPD for the beam splitter configuration. An additional scaling factor is used when considering the polarizer configuration. As the setup is symmetrical for both eyes, crosstalk for the right eye is the same.

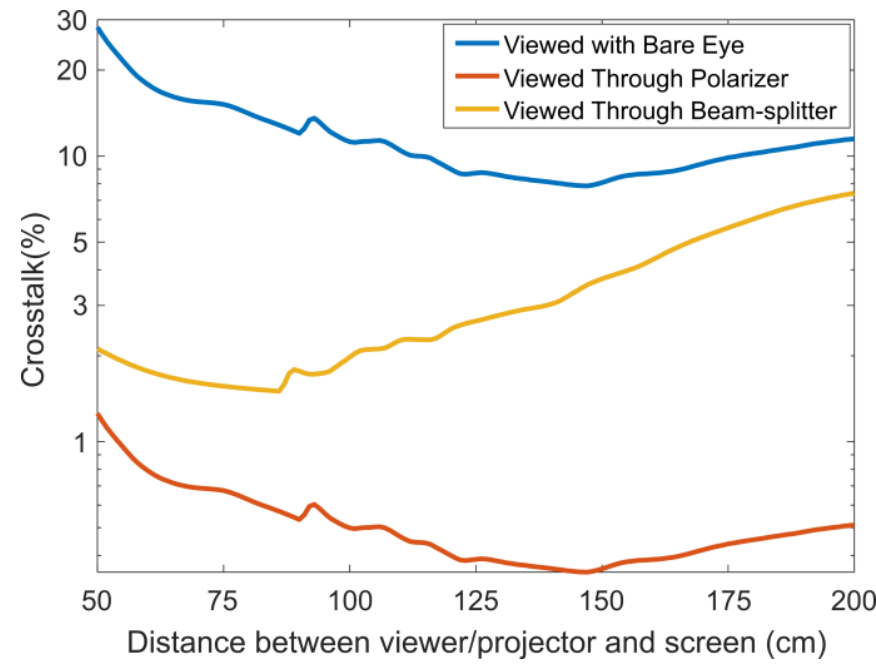

Fig. 7. Amount of stereo crosstalk as a function of screen to viewer distance measured for three eye-projector settings, higher stereo crosstalk is observed when viewing display with bare eyes, while negligible crosstalk when polarizer or beam splitter is placed in front of eye.

Figure 7 shows the crosstalk of the system as function of screen to viewer distance for all three configurations. For bare eye, higher crosstalk is observed at closer distance due to the small eye-box size. The crosstalk is decreased as the viewer moves at the mediocre distance $(125-175 \mathrm{~cm})$ from the screen and increases again at farther distance due to severe eye-box expansion and overlap. The crosstalk is sharply decreased as the mirrors are replaced with beam-splitters and crosstalk of $<5 \%$ is measured for wide range of distance from screen. The minimum crosstalk $(<1 \%)$ is observed when the polarizer film is placed before eye and projector. Since the absorption efficiency of practical 
polarizer film is limited to $98 \%$, the crosstalk in this case is mainly due to the leakage of light in polarizer film.
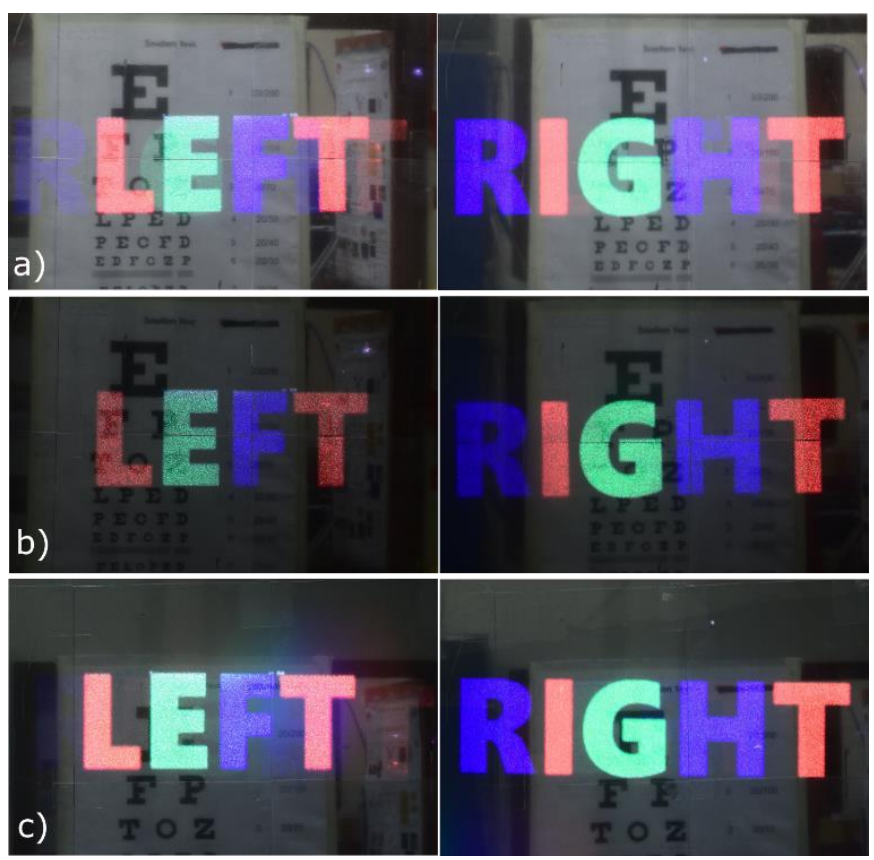

Fig. 8. Shows the left and right views as seen by viewer at $125 \mathrm{~cm}$ from screen and viewed, a) with bare eye, b) through polarizer films and, c) and beam splitters. Same camera settings were used in all the pictures. A printed visual acuity chart on the other side of display can also be seen

Figure 8 shows the photos of left and right views for three eyeprojector configurations captured from the respective eye positions at distance of $125 \mathrm{~cm}$ from the screen and with identical camera settings. As discussed earlier, Fig. 8(a) shows perceptible crosstalk for bare eye configuration, while negligible crosstalk is observed when polarizer (Fig. 8(b)) and beam-splitters (Fig. 8(c)) are used. Additionally, maximum brightness is observed for the beam-splitter due to improved eye projector alignment, while decreased brightness is perceived when polarizers are used.

\section{Brightness and Optical Gain}

The amount of brightness perceived by each eye was measured using experimental values of retro-reflective coefficient $[13,14]$. When a projector illuminates diffused screens, the incident light is scattered in the full hemisphere. On the other hand, a retro-reflective screen provides varying brightness based on the distance between projector and screen and the closeness of eye and projector. The brightness of retro-reflective screen can be measured as [11];

$$
B(d)=C\left(\frac{s}{d}\right) \frac{k \cdot L}{\Omega A}
$$

Where $C(s / d)$ is the value of retro-reflective coefficient at $s / d$ viewing angle, $L$ is the lumen power of projector, $\Omega$ is angle subtended by detector with screen, $A$ is the illuminated screen area and $k$ is the constant for different eye-projector configurations. The values for $k$ are 1, 0.45 and 0.25 for bare eye, polarizer and beam-splitter based configurations, respectively. The optical gain was calculated as the ratio of brightness of the proposed display and regular diffused screen.

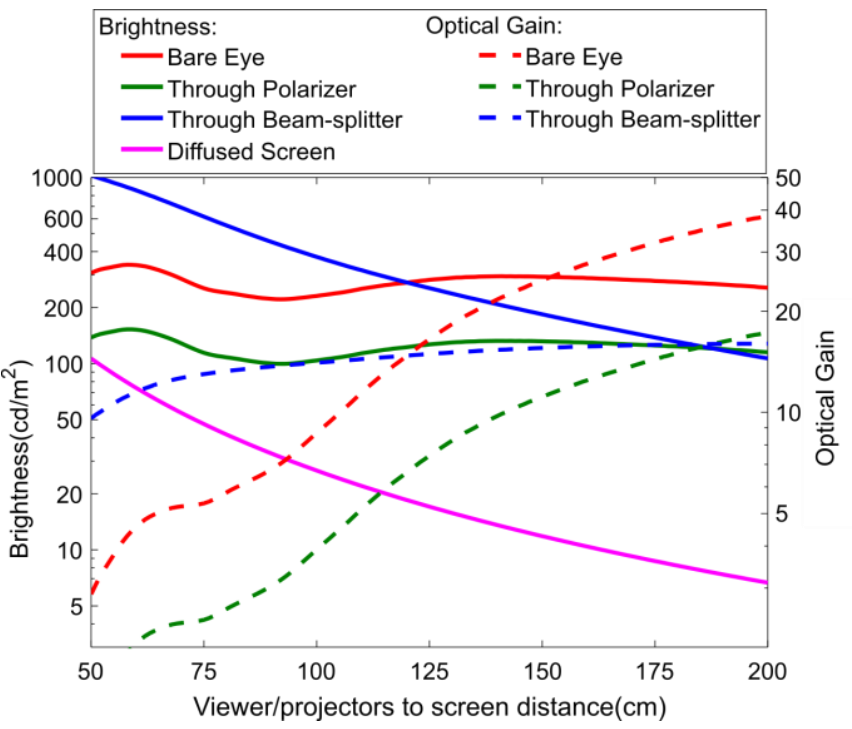

Fig. 9. Shows the brightness and optical gain as the distance between viewer/projector and screen is changed. Maximum brightness with exponential decrease is perceived when eye-projector are aligned using beam-splitter, while nearly constant brightness is perceived when screen is viewed with bare eye or through polarizer. Higher optical gain is observed with bare eye configuration at the farther working distance.

The brightness of the display as a function of screen to viewer distance was obtained by using Eq. (2) and experimentally measured retro-reflective coefficient as shown in Fig. 9. The beam-splitter configuration shows significant brightness (up to $1000 \mathrm{~cd} / \mathrm{m}^{2}$ ) due to the proper alignment of eye with the peak of retro-reflective cone. As the distance increases, the retro-reflective coefficient in Eq. 2 remains constant while illuminated screen area increases with distance and in result the brightness decay is observed in inverse square fashion. For the mirrors and polarizer configurations, the retro-reflective coefficient also changes with the distance and in result almost constant brightness is observed for the range of screen to viewer distance. As the distance increases, the angle $(s / d)$ between eye and associated projector decreases, which moves the angular positon of eye towards the peak of retro-reflective coefficient. For the polarized projector configuration, the additional decrease in the brightness is observed due to the placement of linear polarizer film in front of projectors and eyes. Compared to a diffused screen, the AR display provides 10 times more brightness at closer distance when beam-splitter configuration is used, while the 40 times more brightness is observed at father distance using the bare eye configuration.

\section{CONCLUSION}

We proposed and demonstrated a light efficient augmented reality 3D display using a 75\% see-through retro-reflective screen and a pair of low power mobile projectors. The stereo 3D feature was added by utilizing the retro-reflective property of screen, while the optical seethough capability was added by producing a screen with sparsely patterned retro-reflective microspheres. The retro-reflective and polarization characteristics of the display were experimentally measured and later used to characterize and assess the quality of display. Several viewing configurations (bare eye, polarizers and beamsplitter) were tested, evaluated and compared. Our results showed excellent brightness (up to $1000 \mathrm{~cd} / \mathrm{m}^{2}$ ). The minimum crosstalk (down to $1 \%$ ) was achieved using both beam-splitters and polarizers.

The proposed display is currently limited to fixed arrangement of projectors with respect to viewer's head. The positioning restriction of 
the display can be relaxed by stripping the projector modules and building a head-worn setup. The proposed display can be used for variety of augmented reality applications (i.e. gamming, training/education, medical visualization, 3D head-up-display). The motion parallax can be added by tracking the viewer with respect to screen. Due to the extended working distance and modular assembly of the screen, the display size can be easily increased by using increased number screen segments. The light efficiency and display transparency can also be enhanced by refining the fabrication process.

Funding Information. European Research Council (ERC) under the European Union's Seventh Framework Program (FP7/2007-2013) / ERC advanced grant agreement (340200).

Acknowledgment. We would like to thank Erdem Ulusoy and Sven Holmström for helpful discussions and Vahid Pourreza Ghoushchi for help with capturing setup figures.

\section{References}

1. O. Cakmakci, and J. Rolland. "Head-worn displays: a review." J. Disp. Tech. 2(3), 199-216 (2006).

2. H. Hua, and B. Javidi. "Augmented Reality: Easy on the Eyes." Optics and Photonics News 26(2), 26-33 (2015).

3. Microsoft Hololens, https://www.microsoft.com/microsoft-hololens/enus, accessed December 2016.

4. K. Zhao, and J. Pan. "Optical design for a see-through head-mounted display with high visibility." Opt. Express 24(5), 4749-4760 (2016).

5. J. Wang, X. Xiao, H. Hua, and B. Javidi. "Augmented Reality 3D Displays with Micro Integral Imaging”, J. Disp. Tech. 11(11), 889-893 (2015).
6. K. Hong, J. Yeom, C. Jang, J. Hong, and B. Lee, "Full-color lens-array holographic optical element for three-dimensional optical see-through augmented reality," Opt. Lett. 39(1), 127-130 (2014).

7. M. K. Hedili, M. O. Freeman, and H. Urey, "Transmission characteristics of a bidirectional transparent screen based on reflective microlenses," Opt. Express 21(21), 24636-24646 (2013).

8. K. Saito, and T. Tetsu, "A transparent projection screen based on plasmonic Ag nanocubes." Nanoscale 7(48), 20365-20368 (2015).

9. R. Zhu, H. Chen,T. Kosa, P. Coutino, G. Tan, and S. T. Wu, "High-ambientcontrast augmented reality with a tunable transmittance liquid crystal film and a functional reflective polarizer," J. Soc. Inf. Disp. 24(4), 229-233 (2016).

10. T. Yoshida, S. Kamuro, K. Minamizawa, H. Nii, and S. Tachi, "Repro3d: full-parallax $3 d$ display using retro-reflective projection technology." ACM SIGGRAPH 2010 Emerging Technologies (20).

11. D. Héricz, T. Sarkadi, V. Lucza, V. Kovács, and P. Koppa, "Investigation of a 3D head-mounted projection display using retro-reflective screen," Opt. Express 22(15), 17823-17829 (2014).

12. Z. Rui, and H. Hua. "Imaging quality of a retroreflective screen in headmounted projection displays." JOSA A 26(5), 1240-1249 (2009).

13. S. R. Soomro, and H. Urey. "Design, fabrication and characterization of transparent retro-reflective screen." Opt. Express 24(21), 24232-24241 (2016).

14. S. R. Soomro, and H. Urey. "Augmented Reality 3D Display Using HeadMounted Projectors and Transparent Retro-reflective Screen." Proc. SPIE 10126/1-6 (2017).

15. S. R. Soomro, and H. Urey. "Retro-reflective Characteristics of Transparent Screen for Head Mounted Projection Displays." OSA Frontiers in Optics, FTu5A-2. (2016).

16. Sony pico-projector, http://www.sony.co.in/electronics/projector/mpcl1 (accessed June 2016). 